\title{
Cystic fibrosis drugs: One size does not fit all
}

\section{Steven Molinski and Saumel Ahmadi}

\section{University of Toronto}

Cystic Fibrosis (CF) is the most common autosomal recessive disease, and is caused by mutations in the cystic fibrosis transmembrane conductance regulator (CFTR) gene ${ }^{1}$. CF is a multi-system disease primarily affecting epithelial tissues (e.g. lungs, intestine, pancreas), and the main cause of morbidity and mortality is decreased lung function with age ${ }^{1}$. Pathophysiology of CF is caused by disruption of folding and/or function of the CFTR chloride channel, a membrane protein necessary for maintaining epithelial surface hydration ${ }^{1}$. Importantly, the accepted treatment paradigm involves management of symptoms, while CFTRtargeted therapies are a recent development. However, these drugs are useful in only a small subset of patients.

To date, approximately 2000 CFTR mutations have been identified, and this list continues to grow ${ }^{2}$. F508del is the major mutation, present on at least one allele in $90 \%$ of CF patients (nearly 3600 Canadians), while G551D is second most common ( $5 \%$ of patients, $\sim 200$ Canadians) $^{3}$. Although significant achievements have been made in CF research since the discovery of the CFTR gene at the Hospital for Sick Children in Toronto in 1989, there are still many unanswered questions, and that is why CF remains fatal ${ }^{4}$. However, a major success over the past 50 years in Canada is that the average lifespan of CF patients has increased from 4 to about 50 years (highest in the world) ${ }^{3}$. This is largely attributed to improved clinical care and disease management, although novel CFTR-targeted, drugbased therapies are showing great promise for further enhancement of lifespan and improvement of quality of life in certain patients.

The CF drug Ivacaftor and the G551D mutation: one size fits one size

The first (and only) CFTR mutation-specific drug-based therapy (approved in 2012) repairs the defect of the second most common mutation: G551D. It is a severe gating mutation, which means that it prevents the CFTR chloride channel from opening properly, thereby causing disease ${ }^{5}$. This CFTR-specific therapy, Ivacaftor (also called KalydecoTM or VX-770), acts directly on G551D-CFTR protein to open it and restore normal channel activity ${ }^{5}$. This is only possible because defective G551D-CFTR is in the right place (i.e. properly folded at the cell membrane). Conversely, F508del-CFTR has two defects: a gating defect which can be compared to G551D, as well as a significant folding defect (unlike G551D), so severe that it renders the mutant protein incapable of processing forward to the cell membrane (Figure 1$)^{6}$. Thus, in order to repair F508delCFTR, the trafficking defect must first be overcome. It now becomes clear why Ivacaftor alone showed minimal efficacy in clinical trials on F508del patients :Ivacaftor does not improve processing. Therefore, at least two drugs are needed to repair F508del-CFTR - a corrector that fixes the trafficking defect, and a channel potentiator with properties similar to that of Ivacaftor (Figure 1).

\section{F508del patients do not benefit from Ivacaftor}

Ivacaftor did not improve the health of CF patients with F508del, and for this reason, current research efforts aim to discover novel drugs that improve F508del-CFTR processing. Major leaps forward have been achieved by Vertex Pharmaceuticals in the past decade, where two drug 'hits' from high-throughput screens have proceeded to clinical trials. These compounds, Lumacaftor (VX-809) and VX-661, partially repair F508del-CFTR processing in vitro; however, they do not provide clinical benefit in the presence or absence of Ivacaftor ${ }^{7}$. This can be partly explained by recent evidence suggesting that disease variability between CF patients with the same CFTR mutation (as well as therapeutic responses) is due to the contribution of several modifier genes ${ }^{8}$. Further, it has recently been suggested that Ivacaftor could be detrimental to F508del patients, since it may reduce the quantity and 
A

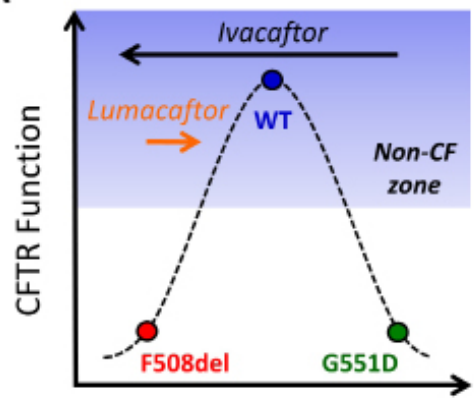

CFTR Stability
B

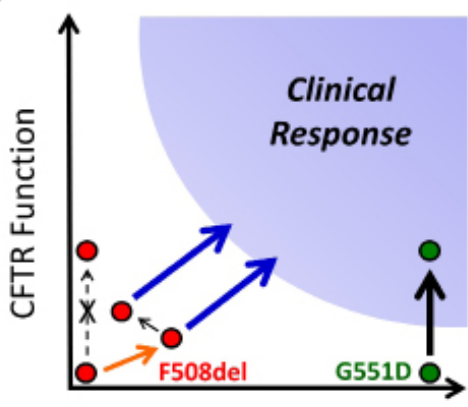

CFTR Quantity

$$
\begin{aligned}
& \rightarrow \text { or } \rightarrow \text { Ivacaftor Treatment } \\
& \rightarrow \text { Lumacaftor Treatment } \\
& \rightarrow \text { Future Treatments }
\end{aligned}
$$

Figure 1. Comparing F508del- and G551DCFTR function, stability and drug responses. (A) Relationship between CFTR function and stability. Wild-type (WT) CFTR has an intermediate stability, allowing for optixmal function, whereas F508del lacks stability (and therefore processing) and G551D is hyperstable (lacks gating activity). Ivacaftor shifts G551D stability toward WT and destabilizes F508del, whereas Lumacaftor increases the stability of F508del, partially rescuing the processing defect. Modified from Cholon et al., 2014.8 (B) CF treatments and their clinical effects on patients with either the F508del or G551D mutation. Lumacaftor partially enhances F508del quantity and function, but Ivacaftor impedes this effect. Therefore, future treatments are required to achieve a clinical response. However, Ivacaftor alone provides a significant clinical response in CF patients with G551D. Modified from Cutting, $2015^{1}$. quality of F508del-CFTR through destabilization (Figure 1) $)^{9}$. Therefore, novel F508del-CFTR-specific drugs must be identified. This may also be the case for other mutations, requiring identification of novel compounds on a case-bycase basis. Unfortunately, it seems that repurposing current drugs is not as simple as initially thought, and previous drug discovery efforts for F508del-CFTR may not be readily translated into therapies for other CFTR mutations.

\section{Path to successful therapies for patients with F508del or more rare mutations}

Understanding drug responses for each CFTR mutation will help elucidate mechanism of action, and assist in the design of mutation-specific therapies (i.e. personalized CF medicine). Ivacaftor and Lumacaftor taught us that repurposing compounds is not straightforward - that one size does not fit all. It is clear that there are future challenges for CF drug discovery. However, there is hope for repurposing certain CF drugs towards mutations within the same dysfunctional class; for example, Ivacaftor is being approved to treat 9 additional CFTR gating mutations ${ }^{10}$. Therefore, by building on this innovation via future clinical testing, it may still be possible to further expand the number of mutations (population size) in which Ivacaftor and Lumacaftor have therapeutic benefits, or in other words, enhance the size in which these drugs fit CF patients.

\section{References}

1. Cutting GR. Cystic fibrosis genetics: from molecular understanding to clinical application. Nat Rev Genet. 2015; 16(1):45-56.
2. Sosnay PR, Siklosi KR, Van Goor F, Kaniecki K, Yu H, Sharma N, Ramalho AS, Amaral MD, Dorfman R, Zielenski J, Masica DL, Karchin R, Millen L, Thomas PJ, Patrinos GP, Corey M, Lewis MH, Rommens JM, Castellani C, Penland CM, Cutting GR. Defining the disease liability of variants in the cystic fibrosis transmembrane conductance regulator gene. Nat Genet. 2013; 45(10):1160-7.

3. Cystic Fibrosis Canada. 2013 Annual Report [internet]. 2013 [updated 2014 April; cited 2015 February 1]. Available from: http://www.cysticfibrosis.ca/ wp-content/uploads/2014/06/CFC_AnnualReport_2013_ENGLISH_FINAL. pdf.

4. Riordan JR, Rommens JM, Kerem B, Alon N, Rozmahel R, Grzelczak Z, Zielenski J, Lok S, Plavsic N, Chou JL, et al. Identification of the cystic fibrosis gene: cloning and characterization of complementary DNA. Science. 1989; 245(4922):1066-73.

5. Van Goor F, Hadida S, Grootenhuis PD, Burton B, Cao D, Neuberger T, Turnbull A, Singh A, Joubran J, Hazlewood A, Zhou J, McCartney J, Arumugam V,Decker C, Yang J, Young C, Olson ER, Wine JJ, Frizzell RA, Ashlock M, Negulescu P. Rescue of CF airway epithelial cell function in vitro by a CFTR potentiator, VX-770. PNAS. 2009; 106(44):18825-30.

6. Molinski S, Eckford PD, Pasyk S, Ahmadi S, Chin S, Bear CE. Functional Rescue of F508del-CFTR Using Small Molecule Correctors. Front Pharmacol. $2012 ; 3: 160$.

7. Okiyoneda T, Veit G, Dekkers JF, Bagdany M, Soya N, Xu H, Roldan A, Verkman AS, Kurth $M$, Simon A, Hegedus T, Beekman JM, Lukacs GL. Mechanism-based corrector combination restores $\triangle$ F508-CFTR folding and function. Nat Chem Biol. 2013; 9(7):444-54.

8. Cholon DM, Quinney NL, Fulcher ML, Esther CR Jr, Das J, Dokholyan NV, Randell SH, Boucher RC, Gentzsch M. Potentiator ivacaftor abrogates pharmacological correction of $\triangle$ F508 CFTR in cystic fibrosis. Sci Transl Med. 2014; 6(246):246ra96.

9. Veit G, Avramescu RG, Perdomo D, Phuan PW, Bagdany M, Apaja PM, Borot F, Szollosi D, Wu YS, Finkbeiner WE, Hegedus T, Verkman AS, Lukacs GL. Some gating potentiators, including VX-770, diminish $\triangle F 508-C F T R$ functional expression. Sci Transl Med. 2014; 6(246):246ra97.

10. Cystic Fibrosis Canada. Patient Group Input to the Common Drug Review at CADTH [internet]. 2014 [updated 2014 July 22; cited 2015 February 1]. Available from: http://www.cysticfibrosis.ca/wp-content/ uploads/2014/07/Patient-Group-Input-Kalydeco-Gating-Mutations22July2014-Final-Version.pdf 


\section{Steven Molinski}

Steven Molinski received his HonBSc (Toxicology) from the University of Toronto, and his MSc (Pharmacology \& Toxicology) from Queen's University under the supervision of Dr. Susan Cole. Currently, he is a PhD candidate (Biochemistry) at the University of Toronto, and works in Dr. Christine Bear's lab group in the Programme of Molecular Structure \& Function at The Hospital for Sick Children. Steven is interested in understanding the molecular basis of human diseases associated with protein misfolding, and has contributed to the working knowledge of Cystic Fibrosis disease in particular.

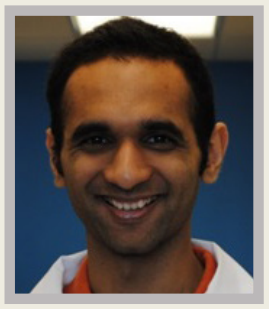

\section{Saumel Ahmadi}

Saumel Ahmadi received his MD from Saurashtra University in India. He is currently a PhD candidate (Physiology) at the University of Toronto, and works in Dr. Christine Bear's lab group in the Programme of Molecular Structure \& Function, as well as Dr. Johanna Rommens' lab group in the Programme of Genetics \& Genome Biology at The Hospital for Sick Children. Saumel is interested in the study of modifier genes and developing novel high-throughput assays for the discovery of Cystic Fibrosis drugs for F508del and other CFTR mutations. 